\title{
VISUALISATION OF DEPENDENCIES BETWEEN CITY STRUCTURE AND THERMAL BEHAVIOUR IN BRNO
}

\author{
J. Novotny ${ }^{\text {a, } * \text {, M. Pivovarnik }}{ }^{\text {a }}$, S. J. Khalsa ${ }^{\text {a }}$, F. Zemek ${ }^{\text {a }}$ \\ ${ }^{a}$ Dept. of Remote Sensing, Global Change Research Institute CAS, Bělidla 986/4a, 60300 Brno, Czech Republic - (novotny.j, \\ pivovarnik.m, khalsa.s, zemek.f)@czechglobe.cz
}

Commission II, ThS 17 - Smart cities

KEY WORDS: Visualization and spatial analysis of urban phenomena, Airborne laser scanning, Hyperspectral thermal imagery, City structure, Thermal regime, Urban heat island.

\begin{abstract}
:
Good understanding of a city's thermal regime and its dependency on the structure of the city provides key knowledge serving as an input for long-term strategic decision-making by local governments. The urban heat island, and more specifically overheating of the streets and adjacent buildings during summer heat waves, has been pointed out as an important issue in the city of Brno, Czech Republic. A complex research effort using remote sensing techniques has started which will analyse the impact of city structure on the thermal behaviour, principally the role of vegetation in the thermal regulation of streets. Two airborne data sets were acquired: hyperspectral data using CASI, SASI and TASI sensors (ITRES, Canada) and lidar mapping using a Riegl 680i instrument (RIEGL, Austria). The thermal data were acquired on two occasions: 7 February 2015 (winter season) and 4 July 2015 (summer season). A laser scanning data-set was acquired on 22 September 2015 with a point cloud density of approximately 15 points $/ \mathrm{m}^{2}$. Surface temperature was retrieved from the thermal hyperspectral data by applying a temperature-emissivity separation algorithm. The 3-D structure of the city was classified from the laser scanning data; we distinguished three main classes: bare land, buildings and vegetation. In the paper we present figures comparing thermal behaviour with other features collected along linear transects through the central part of the city.
\end{abstract}

\section{INTRODUCTION}

The global trend towards increased urbanization, coupled with the impacts of climate change, has generated renewed efforts to understand how the structure and composition of the urban landscape affects local climate. For decades the coarse scale contrast between urban and rural air temperatures, known as the Urban Heat Island (UHI) phenomenon, has been studied using data from sparsely distributed ground level measurements and low resolution satellite sensors (Voogt and Oke, 2003). However, to understand better the relationship between urban temperatures and such factors as vegetation abundance, impervious surface abundance, and geometry of street canyons, data at higher spatial and spectral resolution is needed. This study explores the potential of airborne multi-sensor mapping of an urban area to provide the kinds of detailed information that will make it possible for urban planners to make informed decisions concerning mitigation and adaptation strategies for dealing with increasing urban temperatures.

Inhabitants and stakeholders of Brno, Czech Republic $\left(49^{\circ} 12^{\prime} \mathrm{N}\right.$ $\left.16^{\circ} 37^{\prime} \mathrm{E}\right)$ were interviewed within the UrbanAdapt project (UrbanAdapt, 2016) to discover which effects connected to global change they found most severe. Overheating of the streets in the city was mentioned most often.

The aim of this paper is to visualize the dependencies between city structure and thermal behaviour in Brno; the revealed correlations can serve as an input for modelling of temperature in the city.

\section{METHODS}

\subsection{Study area}

The study was performed using data acquired over the city of Brno. The data examined come one flight line crossing the city from south-west to north-east. These data create a transect encompassing a wide variety of land-cover types. Both ends of the flight line contain rural regions consisting of arable land, meadows and forests. The central part of the flight line includes the old city and the two biggest parks in the city, Lužánky and Špilberk. Old town is surrounded by residential areas which include areas with high density such as blocks of flat-houses and areas with lower density such as family houses. The flight line crosses the Svratka river as well.

Data were acquired in both winter and summer seasons in order to cover both climatological extremes. Winter acquisition was performed on 7th February 2015 at 21:53 (UTC). Summer acquisition was performed on 4th July 2015 at 14:03 (UTC) and at 20:59 (UTC).

Complementary airborne laser scanning dataset was acquired on 22nd September 2015.

\footnotetext{
* Corresponding author
} 


\subsection{Remotely sensed data}

The Flying Laboratory of Imaging Systems (FLIS) (Hanuš et al, 2014) operated by Global Change Research Institute CAS (Brno, Czech Republic) was used for data acquisition. FLIS consists of Compact Airborne Spectrographic Imager (CASI), Shortwave infrared Airborne Spectrographic Imager (SASI) and Thermal Airborne Spectrographic Imager (TASI). All sensors are developed by Itres Ltd. (Calgary, Canada).

CASI sensor acquired data in the spectral range of 370-1040 nm in 48 spectral bands. Full Width at Half Maximum (FWHM) of each of its bands was $14 \mathrm{~nm}$. SASI sensor operated in spectral range of $960-2440 \mathrm{~nm}$ covered by 100 spectral bands with $\mathrm{FWHM} \approx 15 \mathrm{~nm}$. TASI sensor data contained 32 bands situated in the $8-11.5 \mu \mathrm{m}$ region and have a FWHM $\approx 0.11 \mu \mathrm{m}$.

Airborne laser scanning was performed with a Riegl Q680i instrument operated by a TopGis company. It is a full-waveform (FWF) scanner operating at $1550 \mathrm{~nm}$. Waveform decomposition and geometrical correction were performed by a vendor using RiProcess software. The data were delivered in LAS format after a strip adjustment, providing 3D coordinates and two basic FWF variables - amplitude and pulse width - for each point. The final cloud density was approximately 15 points per square meter.

\subsection{Image processing}

Data acquired by CASI, SASI and TASI sensor were radiometrically and atmospherically corrected and georeferenced to UTM coordinate system. Radiometric corrections were performed according to laboratory and inflight sensor calibrations. Atmospheric corrections of CASI and SASI sensor were performed with ATCOR-4 Ver. 6.0 (Richter, 2012), which takes into account supporting field measurements. These include reflectance measurement of calibration targets with FielSpec4 (ASD, Inc. U.S.) and measurement of aerosol and water vapour content with sunphotometer CIMEL (Cimel Electronique S. A. S). Atmospheric corrections of TASI sensor are based on results of MODerate resolution atmospheric TRANsmission (MODTRAN) (Berk et al, 2006). Atmospheric temperature and water vapour profiles, as inputs to MODTRAN, were obtained from MODIS product MOD07_L2 (Borbas et al, 2011).

Temperature and emissivity were estimated by the temperatureemissivity separation algorithm (TES), which was originally developed for ASTER sensor (Gillespie et al, 1998). It is based on semi-empirical relationship between spectral contrast of emissivity (i.e. highest and lowest values of emissivity spectrum) and minimum value of spectral emissivity $\varepsilon_{\min }$. The relationship is expressed in the following:

$$
\varepsilon_{\text {min }}=1.001-0.737 \mathrm{MMD}^{0.760},
$$

where MMD stands for Maximum-Minimum emissivity Difference. The coefficients in (1) were derived from regression on 108 spectra of natural materials chosen from ASTER spectral library (Baldridge et al, 2009). These coefficients were determined with respect to TASI response functions.

Lidar data processing was done in ENVI Lidar software. Ground filtering was the first step, followed by an interpolation of digital terrain model (DTM) and digital surface model (DSM). The first model is used for derivation of height above sea level, slope and orientation variables. We obtained height of above-ground objects subtracting DSM-DTM. Above-ground points were classified then into two principal classes - (high) vegetation and buildings.

The digital surface model was used to compute direct plus diffuse solar irradiation accumulated during the daylight hours of 4th July 2015. The computation was done using the SAGA Lighting and Visibility module (SAGA, 2013). Surface absorptivity was estimated by subtracting from one the average surface reflectance over the visible bands of the CASI sensor. In Fig. 1 and Fig. 2 the product of solar irradiation and absorptivity is given as a measure of the amount of solar energy absorbed by the surface.

\subsection{Visualisation of dependencies between structure and temperature}

We use a transect approach to depict the dependencies between the structure of the city and the surface temperature. We generated several rasters at a common spatial resolution. The first three rasters have surface temperature from the three flight campaigns - winter night $\left(\mathrm{T}_{\mathrm{W}}\right)$, summer day $\left(\mathrm{T}_{\mathrm{D}}\right)$ and summer night $\left(\mathrm{T}_{\mathrm{N}}\right)$. The second set of rasters depicts the structure of the city. There are two rasters with a digital surface model, a digital terrain model and a raster with a prevailing classification of all points inside each pixel. The third set of rasters depicts characteristics of the surface. NDVI vegetation index was calculated from NIR and RED bands of the CASI data and a raster of absorbed energy was calculated as a product of solar irradiation and absorptivity, as described in the previous section.

With two transects overlaying the area of interest we collected values from 1000 sampling points on each, where each sampling point represents a median value from a circle with a radius of 1 meter.

Sampled data were visualised using ENVI (Exelis VIS) and Mathematica (Wolfram Research) and are displayed in Figures 1 and 2.

\section{RESULTS}

Results of visualisation are presented in Figure 1 and Figure 2. Both figures have the following structure. A distance along the transect in meters forms a common $\mathrm{X}$ axis. A true-colour composition from CASI data is shown in the topmost panel, the transect is drawn with a yellow line. Surface temperatures of a summer day and a summer night are shown in the second panel. Surface temperature of a winter night is shown in the third panel. A depiction of city structure is contained in the fourth panel. Terrain level is shown in brown, while buildings are distinguished from high vegetation with grey and green colours, respectively. NDVI is shown in fifth panel and absorbed energy is shown in the last panel.

Several common observations can be made in both figures. NDVI as a measure of "greenness" follows a classification of high vegetation and also allows distinguishing between streets and a surface covered by grass. Local minima in absorbed energy follow shaded regions at the edges of buildings. Temperature over areas covered by vegetation tends to be more stable and lower in average, while the temperature of streets and parking lots is significantly higher during summer day. 


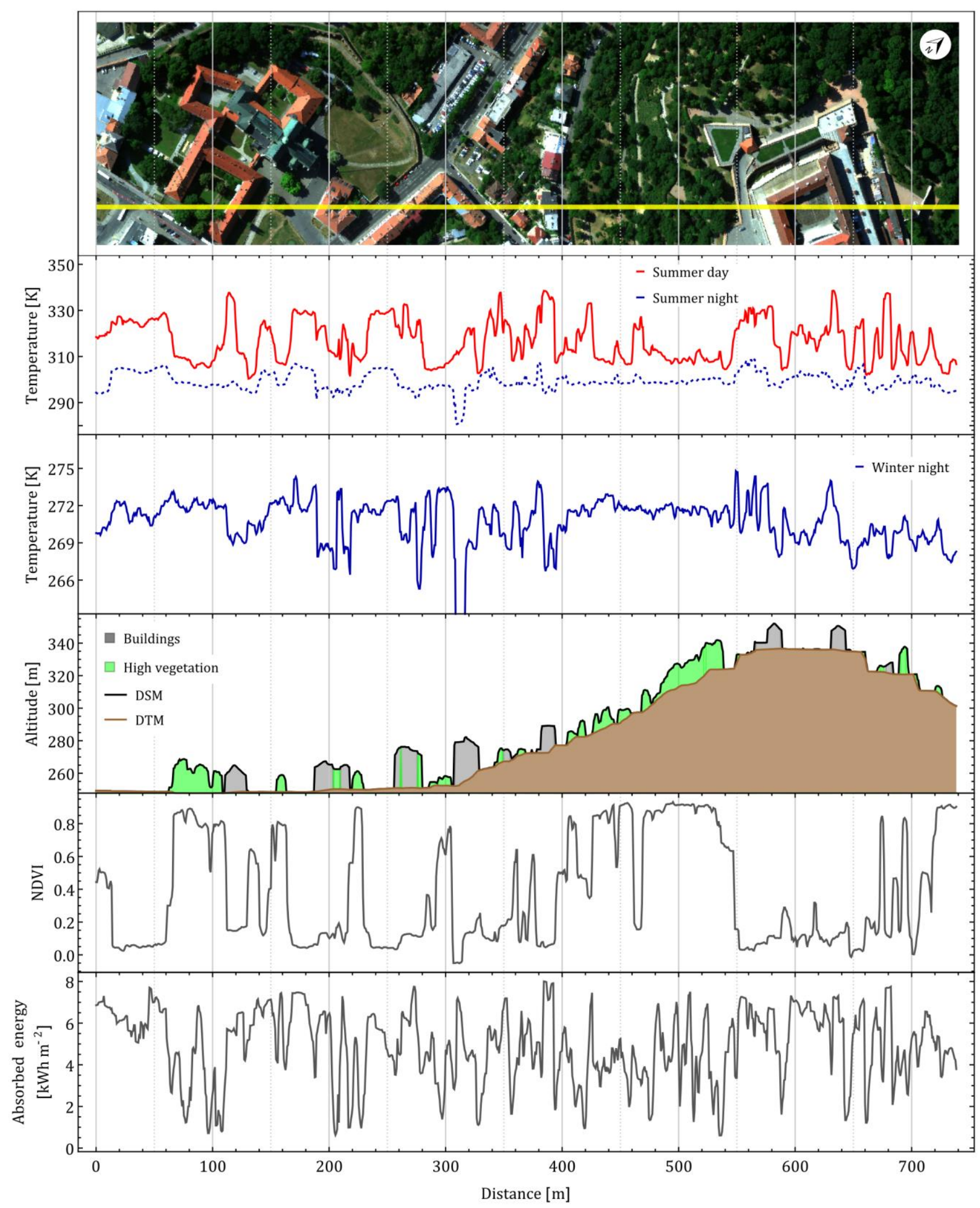

Figure 1. Visualisation of transect 1. For a detailed explanation, please, see the section Results. 


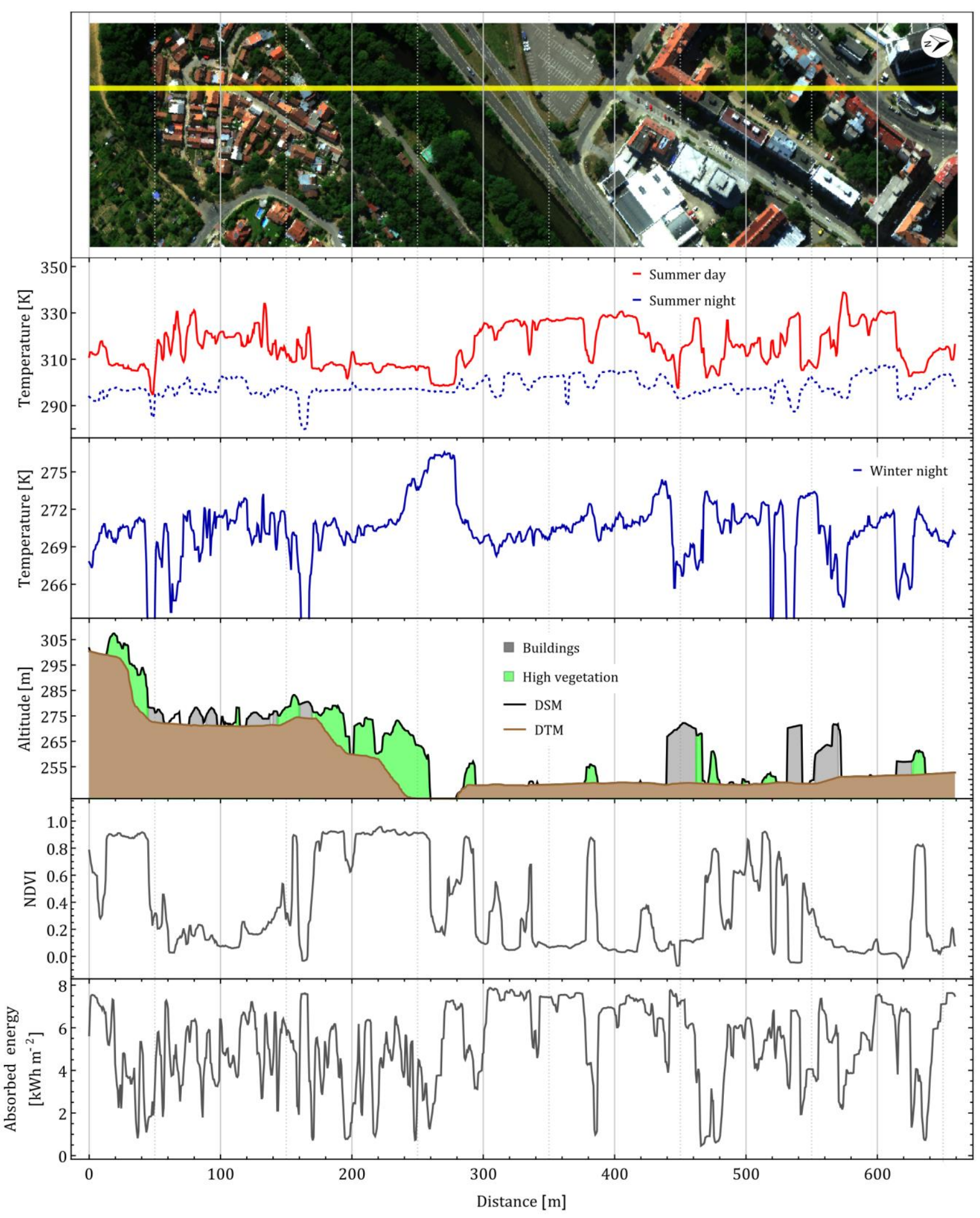

Figure 2. Visualisation of transect 2. For a detailed explanation, please, see the section Results. 
We would like to point out several interesting features in individual transects. We will use an $\mathrm{X}$ axis to label the details.

In Figure 1 between 80 and 90, the stabilising role of vegetation can be seen - the temperature profile has a low variability as well as a lower average despite a higher amount of absorbed energy. In Figure 1 at 305 and 390, two different roof surfaces can be observed - the rightmost one is dark, has a high absorptivity, while the leftmost one has a high reflectivity and reflects a cold sky in both summer and winter night. The region from 400 to 550 contains green areas which surrounds Špilberk castle. Summer day temperature is significantly lower in this region compared to other built up areas. The only notable temperature extreme is visible between 460 and 470 where the transect crosses a path walk.

Figure 2 shows interesting features as well. Roofs with high reflectivity can be observed at $160-170$ and $530-540$. The coldest places during a summer day are the river between 260 and 280 (which is on the other hand the warmest place in winter) and hard shadows next to high buildings, e.g. at 545 . There is a notable shaded hillside between 40 and 50 causing temperature decrease in both summer day and night. There is an interesting dip in the summer night temperature profile between 360 and 370 , which is presumably caused by a parked car in the parking lot. A dip in the summer temperature around 445 is caused by a roof window included in the transect.

\section{CONCLUSIONS}

In this paper we showed remotely sensed data with a high spatial resolution can be a valuable input for a visualisation of dependencies between city structure and a thermal regime. The data also allows us to quantify the correlations, e.g. the effect of vegetation on a street temperature. Modelling such relationships will be a future continuation in the project.

\section{ACKNOWLEDGEMENTS}

The authors would like to thank Jan Hanuš, Tomáš Fabiánek and Lukáš Fajmon for processing of hyperspectral data, and Marty Petach for processing the solar illumination data.

This work was supported by the Ministry of Education, Youth and Sports of CR within the CzeCOS program, grant number LM2015061 and within the National Sustainability Program I (NPU I), grant number LO1415. Other funding came from Norwegian funds via UrbanAdapt project EHP-CZ02-OV-1036-2015.

\section{REFERENCES}

Baldridge, A. M., Hook, S. J., Grove, C. I., and Rivera G., 2009. The ASTER spectral library version 2.0. Remote Sensing of Environment, vol. 113, no. 4, pp. 711-715.

Berk, A., Anderson, G.P., Acharya, P.K., Bernstein, L.S., Muratov, L., Lee, J., Fox, M., Adler-Golden, S.M., Chetwynd, J.H., Hoke, M.L., Lockwood, R.B., Gardner, J.A., Cooley, T. W., Borel, C.C., Lewis, P.E., and Shettle, E.P., 2006. MODTRAN (TM) 5: 2006 update - art. no. 62331f. Proceedings of the society of photo-optical instrumentation engineers (SPIE), 6233, pp. F2331-F2331,

Borbas, E.E., Seemann, S.W., Kern A., Moy L., Li J., Gumley L., and Menzel W.P, 2011. MODIS Atmospheric Profile Retrieval - ATBD, Madison, WI 53706, United States, http://modis-

atmos.gsfc.nasa.gov/_docs//MOD07_atbd_v7_April2011.pdf .

Gillespie, A., Rokugawa, S., Matsunaga, T., Cothern J., Hook, S., and Kahle, A., 1998. A temperature and emissivity separation algorithm for Advanced Spaceborne Thermal Emission and Reflection Radiometer (ASTER) images. IEEE Transactions on Geoscience and Remote Sensing, 36(4), pp. 1113-1126.

Hanuš, J., Fabiánek, T., Kaplan, V., and Homolová, L, 2014. Flying laboratory of imaging systems (FLIS) at CzechGlobe. SGEM2014 Conference Proceedings, pp. 177-182.

Richter, R. 2012. Atmospheric / topographic correction for airborne imagery (ATCOR 4 user guide, version 6.2 BETA). Tech. rep., DLR - German Aerospace Center, Wessling, Germany.

SAGA, GIS, 2013. "System for automated geoscientific analyses." Available at: http://www.saga-gis.org/.

UrbanAdapt, 2016. "Urban adaptation to climate change" Available at: http://urbanadapt.cz/en.

Voogt, J A, and T R Oke, 2003. Thermal Remote Sensing of Urban Climates. Remote Sensing of Environment, 86 (3): pp. $370-8$ 